\title{
Modelo Analítico de um Algoritmo LMS de Passo Variável Baseado na Autocorrelação do Sinal de Erro
}

\author{
José Gil Fausto Zipf, Orlando José Tobias e Rui Seara
}

\begin{abstract}
Resumo-Algoritmos adaptativos de passo variável têm uma significativa importância prática, na medida em que apresentam um melhor desempenho em relação aos algoritmos de passo fixo. A literatura técnica é vasta nesse assunto, resultando em uma área de pesquisa bastante ativa. Uma das principais estratégias de ajuste do passo de adaptação considera a função de autocorrelação do sinal de erro, a qual deu origem ao algoritmo LMS de passo variável proposto por Aboulnasr. $O$ presente artigo propõe desenvolver um modelo analítico deste bem conhecido algoritmo da literatura. Particularmente, são derivados modelos estocásticos para o comportamento médio do vetor de coeficientes e para a curva de aprendizagem. Simulações numéricas confirmam a precisão do modelo proposto.
\end{abstract}

Palavras-chave-Algoritmos LMS de passo variável, autocorrelação do sinal de erro, modelagem de filtros adaptativos.

Abstract-Variable step-size adaptive algorithms have significant practical importance, since they present better performance as compared with the ones having fixed step size. The technical literature is vast in such a subject, resulting in a very active research area. One of the main strategies for adjusting the step size considers the error signal autocorrelation function, which gave rise to the variable step-size LMS algorithm proposed by Aboulnasr. The present paper proposes to develop an analytical model of this well-known algorithm from the literature. In particular, stochastic models for the mean weight behavior and the learning curve are derived. Numerical simulation results verify the accuracy of the proposed model.

Keywords-Variable step-size LMS algorithms, error signal autocorrelation, modeling of adaptive filters.

\section{INTRODUÇÃO}

Os algoritmos LMS são os mais utilizados em aplicações de filtragem adaptativa, devido principalmente à sua simplicidade de implementação e robustez nos mais diversos cenários. [1]. O algoritmo LMS convencional opera com um passo de adaptação fixo, selecionado a partir de um

José Gil F. Zipf, Orlando J. Tobias e Rui Seara, LINSE - Laboratório de Circuitos e Processamento de Sinais, Depto. de Eng. Elétrica, Universidade Federal de Santa Catarina, Florianópolis, SC, E-mails: \{gil, orlando, seara\}@linse.ufsc.br.

José Gil F. Zipf e Orlando J. Tobias são também Professores do Departamento de Engenharia Elétrica e Telecomunicações da Universidade Regional de Blumenau (FURB), Blumenau, SC, Brasil.

Este trabalho foi parcialmente financiado pelo Conselho Nacional de Desenvolvimento Científico e Tecnológico (CNPq). compromisso existente entre velocidade de convergência e desajuste. Com o propósito de se obter simultaneamente rápida convergência e reduzido desajuste, surgem os algoritmos LMS de passo variável (variable step-size LMS VSSLMS). Atualmente, na literatura, existem diferentes estratégias de algoritmos VSSLMS, cada uma delas propondo diferente forma de ajuste do passo de adaptação. As aplicações dos algoritmos VSSLMS são as mesmas do algoritmo LMS convencional, a saber: identificação de sistema, cancelamento de ruído, equalização de canal, dentre outras [1]. A idéia central desses algoritmos consiste em se utilizar um passo de adaptação de valor elevado no início da fase de aprendizagem e gradualmente reduzi-lo, conforme se aproxime a fase de regime permanente.

Nos últimos anos, muitos autores propuseram diferentes formas de atualização do passo de adaptação variável [2]-[21]. Em [9], é discutido um algoritmo VSSLMS baseado no sinal de erro ao quadrado, aqui denominado algoritmo de Kwong. Tal algoritmo exibe um satisfatório desempenho para a maioria das aplicações, apresentando contudo uma forte dependência do processo de ajuste do passo variável e do desajuste com respeito ao ruído de medição. Uma modificação no algoritmo de Kwong é apresentada em [12], resultando no denominado algoritmo de Aboulnasr. Este algoritmo melhora consideravelmente a imunidade ao ruído branco de medição, na medida em que considera a função de autocorrelação do sinal de erro no processo de atualização do passo de adaptação. No entanto, em nosso conhecimento, não há na literatura qualquer modelo analítico que possibilite uma predição de desempenho do algoritmo de Aboulnasr. Tal modelo poderia auxiliar em uma melhor compreensão e melhoria do algoritmo em diversas aplicações. Neste trabalho de pesquisa, é apresentado um modelo estocástico do algoritmo de Aboulnasr, determinando expressões analíticas para o comportamento médio do vetor de coeficientes e para a curva de aprendizagem. Resultados de simulação numérica, considerando um problema de identificação de sistema, comprovam a precisão do modelo proposto.

\section{Algoritmo DE KWONG}

Considere um problema de identificação de sistema ilustrado pelo diagrama de blocos da Fig. 1. Nessa figura, a saída do sistema desconhecido é 


$$
d(n)=\mathbf{w}_{\mathrm{o}}^{\mathrm{T}} \mathbf{x}(n)+\eta(n)
$$

onde $\mathbf{x}(n)=[x(n) x(n-1) \cdots x(n-N+1)]^{\mathrm{T}}$ denota o vetor de entrada, assumindo $\{x(n)\}$ um processo gaussiano de média zero e variância $\sigma_{x}^{2}, \quad \mathbf{w}(n)=\left[w_{0}(n) w_{1}(n) \cdots w_{N-1}(n)\right]^{\mathrm{T}}$ é o vetor de coeficientes do filtro adaptativo, $\mathbf{w}_{0}=\left[\begin{array}{lll}w_{0} & w_{1} & \cdots\end{array}\right.$ $\left.w_{N-1}\right]^{\mathrm{T}}$ representa a resposta ao impulso do sistema a ser identificado e $\eta(n)$ é um ruído de medição i.i.d. com média nula e variância $\sigma_{\eta}^{2}$. O sinal de erro é dado por

$$
e(n)=d(n)-\mathbf{w}^{\mathrm{T}}(n) \mathbf{x}(n)
$$

A expressão de atualização do vetor de coeficientes para o algoritmo VSSLMS pode ser escrita como

$$
\mathbf{w}(n+1)=\mathbf{w}(n)+\mu(n) e(n) \mathbf{x}(n)
$$

onde $\mu(n)$ é o passo variável de adaptação.

No algoritmo de Kwong, o sinal de erro ao quadrado é utilizado para atualizar o passo de adaptação variável de acordo com a seguinte expressão:

$$
\mu(n+1)=\alpha \mu(n)+\gamma e^{2}(n)
$$

onde $\alpha$ e $\gamma$ são parâmetros positivos de controle.

A principal motivação para este algoritmo é que quanto maior for o erro de predição, maior deve ser o valor do passo de adaptação, de maneira a acelerar o processo de convergência. Por outro lado, à medida que o erro diminui, o valor do passo de adaptação também deve ser reduzido, com o objetivo de se obter um menor desajuste [9]. Um problema desse algoritmo é a sua forte dependência com ruído de medição, tendo seu desempenho bastante deteriorado em ambientes com baixa razão sinal-ruído (SNR).

\section{Algoritmo de AboulnasR}

O algoritmo de Aboulnasr pode ser visto como uma modificação do algoritmo de Kwong na medida em que utiliza a autocorrelação entre $e(n)$ e $e(n-1)$, ao invés do sinal de erro ao quadrado $e^{2}(n)$, para atualização do passo variável de adaptação. Dessa forma, o algoritmo pode efetivamente manter uma razoável imunidade ao ruído de medição não correlacionado. Para atualizar o passo de adaptação, a estratégia de Aboulnasr [12] considera para ajuste o quadrado da estimativa da autocorrelação do sinal de erro. Tal estimativa (obtida através de uma filtragem passa-baixas) é dada por

$$
p(n)=\beta p(n-1)+(1-\beta) e(n) e(n-1)
$$

onde $\beta>0$ é um parâmetro de controle. $O$ ajuste do passo de adaptação é realizado conforme a seguinte expressão:

$$
\mu(n+1)=\alpha \mu(n)+\gamma p^{2}(n)
$$

onde, novamente, $\alpha$ e $\gamma$ são parâmetros positivos de controle.
Para garantir uma operação estável do algoritmo adaptativo, os limites de ajuste do passo de adaptação são fixados pela seguinte inequação [1]:

$$
0<\mu(n)<\frac{2}{3 \operatorname{tr}[\mathbf{R}]} .
$$

\section{MODELAGEM}

A modelagem do algoritmo de Aboulnasr [12] é derivada invocando parcialmente a Teoria da Independência (TI) e assumindo as seguintes hipóteses simplificativas:

i) O passo variável de adaptação $\mu(n)$ é independente e descorrelacionado de qualquer outro sinal no sistema.

ii) $E\left[\mu^{2}(n)\right]=E^{2}[\mu(n)]$.

iii) $E[\mu(n) \mu(n-1)]=E[\mu(n)] E[\mu(n-1)]$.

iv) $E\left[p^{2}(n)\right]=E^{2}[p(n)]$

As hipóteses acima são válidas sob condições de adaptação lenta do passo $\mu(n)$.

\section{A. Comportamento Médio do Vetor de Coeficientes}

O modelo para o comportamento médio do vetor de coeficientes é obtido tomando o valor esperado de (3) e considerando a hipótese (i). Então,

$$
E[\mathbf{w}(n+1)]=E[\mathbf{w}(n)]+E[\mu(n)]\{\mathbf{p}-\mathbf{R} E[\mathbf{w}(n)]\}
$$

onde $\mathbf{R}=E\left[\mathbf{x}(n) \mathbf{x}^{\mathrm{T}}(n)\right]$ é a matriz de autocorrelação do vetor de entrada e $\mathbf{p}=E[d(n) \mathbf{x}(n)]$ representa o vetor de correlação cruzada entre o sinal desejado e o vetor de entrada. O valor esperado $E[\mu(n)]$ deve ser determinado em (8). Assim, tomando o valor esperado de ambos os lados de (6), obtém-se

$$
E[\mu(n+1)]=\alpha E[\mu(n)]+\gamma E\left[p^{2}(n)\right] .
$$

Assumindo adaptação lenta e considerando (iv), (9) pode ser reescrita como

$$
E[\mu(n+1)]=\alpha E[\mu(n)]+\gamma E^{2}[p(n)] .
$$

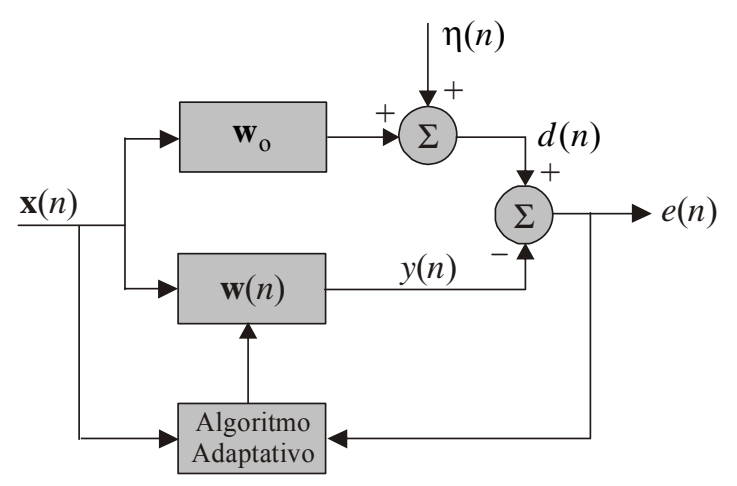

Fig. 1. Diagrama de blocos de um esquema de identificação de sistema. 
Com o objetivo de se determinar $E[p(n)]$ em (10), toma-se o valor esperado de ambos os lados de (5). Assim,

$$
E[p(n)]=\beta E[p(n-1)]+(1-\beta) E[e(n) e(n-1)]
$$

Logo, $E[e(n) e(n-1)]$ precisa agora ser obtido em (11). Para tal, considere o vetor de erro nos coeficientes dado por

$$
\mathbf{v}(n)=\mathbf{w}(n)-\mathbf{w}_{\mathrm{o}} .
$$

Dessa forma, os sinais de erro $e(n)$ e $e(n-1)$ podem ser escritos, respectivamente, como

$$
e(n)=\eta(n)-\mathbf{v}^{\mathrm{T}}(n) \mathbf{x}(n)
$$

e

$$
e(n-1)=\eta(n-1)-\mathbf{v}^{\mathrm{T}}(n-1) \mathbf{x}(n-1) .
$$

Agora, considerando (13) e (14), pode-se escrever

$$
\begin{aligned}
e(n) e(n-1)= & \eta(n) \eta(n-1)-\eta(n) \mathbf{v}^{\mathrm{T}}(n-1) \mathbf{x}(n-1) \\
& -\eta(n-1) \mathbf{v}^{\mathrm{T}}(n) \mathbf{x}(n) \\
& +\mathbf{v}^{\mathrm{T}}(n) \mathbf{x}(n) \mathbf{x}^{\mathrm{T}}(n-1) \mathbf{v}(n-1) .
\end{aligned}
$$

Então, tomando o valor esperado de ambos os lados de (15), tem-se

$$
E[e(n) e(n-1)]=E\left[\mathbf{v}^{\mathrm{T}}(n) \mathbf{x}(n) \mathbf{x}^{\mathrm{T}}(n-1) \mathbf{v}(n-1)\right] .
$$

Agora, definindo a matriz de correlação entre os vetores $\mathbf{x}(n)$ e $\mathbf{x}(n-1)$ como

$$
\mathbf{R}_{1}=E\left[\mathbf{x}(n) \mathbf{x}^{\mathrm{T}}(n-1)\right]
$$

a expressão (16) pode ser então reescrita como

$$
E[e(n) e(n-1)]=E\left[\mathbf{v}^{\mathrm{T}}(n) \mathbf{R}_{1} \mathbf{v}(n-1)\right] .
$$

Note que $\mathbf{R}_{1}$ é obtida sem considerar a TI, uma vez que de acordo com tal hipótese $E\left[\mathbf{x}(n) \mathbf{x}^{\mathrm{T}}(n-1)\right]=0$.

Então, definindo

$$
\mathbf{K}_{1}(n)=E\left[\mathbf{v}(n) \mathbf{v}^{\mathrm{T}}(n-1)\right]
$$

a expressão (18) pode agora ser reescrita como segue:

$$
E[e(n) e(n-1)]=\operatorname{tr}\left[\mathbf{R}_{1} \mathbf{K}_{1}(n)\right] .
$$

Para se obter o modelo desejado, é necessário estimar $\mathbf{R}_{1} \mathrm{e}$ determinar uma expressão para $\mathbf{K}_{1}(n)$. Assim, substituindo (12) em (3), a expressão de atualização do vetor de erro nos coeficientes pode ser escrita como

$$
\mathbf{v}(n+1)=\mathbf{v}(n)+\mu(n) e(n) \mathbf{x}(n) .
$$

Representando o sinal de erro como função de $\mathbf{v}(n)$, obtém-se

$$
\begin{aligned}
e(n) & =d(n)-\left[\mathbf{x}^{\mathrm{T}}(n) \mathbf{w}(n)\right] \\
& =d(n)-\mathbf{x}^{\mathrm{T}}(n) \mathbf{v}(n)-\mathbf{x}^{\mathrm{T}}(n) \mathbf{w}_{\mathrm{o}} .
\end{aligned}
$$

Substituindo agora (22) em (21), tem-se

$$
\begin{aligned}
\mathbf{v}(n+1)= & \mathbf{v}(n)+\mu(n)[d(n) \mathbf{x}(n) \\
& \left.-\mathbf{x}(n) \mathbf{x}^{\mathrm{T}}(n) \mathbf{v}(n)-\mathbf{x}(n) \mathbf{x}^{\mathrm{T}}(n) \mathbf{w}_{\mathrm{o}}\right]
\end{aligned}
$$

e desde que

$$
d(n)-\mathbf{x}^{\mathrm{T}}(n) \mathbf{w}_{\mathrm{o}}=\eta(n)
$$

pode-se escrever

$$
\mathbf{v}(n+1)=\mathbf{v}(n)+\mu(n)\left[\eta(n) \mathbf{x}(n)-\mathbf{x}(n) \mathbf{x}^{\mathrm{T}}(n) \mathbf{v}(n)\right]
$$

e

$$
\begin{aligned}
\mathbf{v}^{\mathrm{T}}(n)= & \mathbf{v}^{\mathrm{T}}(n-1)+\mu(n-1)\left[\eta(n-1) \mathbf{x}^{\mathrm{T}}(n-1)\right. \\
& \left.-\mathbf{v}^{\mathrm{T}}(n-1) \mathbf{x}(n-1) \mathbf{x}^{\mathrm{T}}(n-1)\right] .
\end{aligned}
$$

Então, fazendo o produto $\mathbf{v}(n+1) \mathbf{v}^{\mathrm{T}}(n)$, chega-se a

$$
\begin{aligned}
& \mathbf{v}(n+1) \mathbf{v}^{\mathrm{T}}(n)= \\
& \mathbf{v}(n) \mathbf{v}^{\mathrm{T}}(n-1) \\
& +\mu(n-1) \eta(n-1) \mathbf{v}(n) \mathbf{x}^{\mathrm{T}}(n-1) \\
& -\mu(n-1) \mathbf{v}(n) \mathbf{v}^{\mathrm{T}}(n-1) \mathbf{x}(n-1) \mathbf{x}^{\mathrm{T}}(n-1) \\
& +\mu(n) \eta(n) \mathbf{x}(n) \mathbf{v}^{\mathrm{T}}(n-1) \\
& -\mu(n) \mathbf{x}(n) \mathbf{x}^{\mathrm{T}}(n) \mathbf{v}(n) \mathbf{v}^{\mathrm{T}}(n-1) \\
& +\mu(n) \mu(n-1) \eta(n) \eta(n-1) \mathbf{x}(n) \mathbf{x}^{\mathrm{T}}(n-1) \\
& -\mu(n) \mu(n-1) \eta(n) \mathbf{x}(n-1) \mathbf{x}^{\mathrm{T}}(n-1) \mathbf{x}(n) \mathbf{v}^{\mathrm{T}}(n-1) \\
& -\mu(n) \mu(n-1) \eta(n-1) \mathbf{x}(n) \mathbf{x}^{\mathrm{T}}(n) \mathbf{v}(n) \mathbf{x}^{\mathrm{T}}(n-1) \\
& +\mu(n) \mu(n-1) \mathbf{x}(n) \mathbf{x}^{\mathrm{T}}(n) \mathbf{v}(n) \mathbf{v}^{\mathrm{T}}(n-1) \mathbf{x}(n-1) \mathbf{x}^{\mathrm{T}}(n-1) .
\end{aligned}
$$

Agora, tomando o valor esperado de (27), obtém-se

$$
\begin{aligned}
\mathbf{K}_{1}(n+1)= & \mathbf{K}_{1}(n)-E[\mu(n)] E\left[\mathbf{x}(n) \mathbf{x}^{\mathrm{T}}(n) \mathbf{v}(n) \mathbf{v}^{\mathrm{T}}(n-1)\right] \\
& -E[\mu(n-1)] E\left[\mathbf{v}(n) \mathbf{v}^{\mathrm{T}}(n-1) \mathbf{x}(n-1) \mathbf{x}^{\mathrm{T}}(n-1)\right] \\
& +E[\mu(n) \mu(n-1)]\left[\mathbf{x}(n) \mathbf{x}^{\mathrm{T}}(n) \mathbf{v}(n) \mathbf{v}^{\mathrm{T}}(n-1)\right. \\
& \left.\times \mathbf{x}(n-1) \mathbf{x}^{\mathrm{T}}(n-1)\right] .
\end{aligned}
$$

Finalmente, desenvolvendo (28) sob a luz da hipótese (iii) e considerando o teorema de fatoração de momentos para sinais gaussianos [1], tem-se

$$
\begin{aligned}
\mathbf{K}_{1}(n+1)= & \mathbf{K}_{1}(n)-E[\mu(n)] \mathbf{R} \mathbf{K}_{1}(n)-E \mu(n-1) \mathbf{K}_{1}(n) \mathbf{R} \\
& +E[\mu(n)] E[\mu(n-1)]\left\{\mathbf{R}_{1} \operatorname{tr}\left[\mathbf{R}_{1} \mathbf{K}_{1}(n)\right]\right. \\
& +\mathbf{R K}_{1}(n) \mathbf{R}+\mathbf{R}_{1} \mathbf{K}_{1}(n) \mathbf{R}_{1}
\end{aligned}
$$

para $\mathbf{R}=E\left[\mathbf{x}(n) \mathbf{x}^{\mathrm{T}}(n)\right]$

\section{B. Curva de Aprendizagem}

Agora, utilizando (25), pode-se encontrar uma expressão recursiva que descreva a evolução $\operatorname{de} \mathbf{v}(n) \mathbf{v}^{\mathrm{T}}(n)$. Então, 


$$
\begin{aligned}
\mathbf{v}(n+1) \mathbf{v}^{\mathrm{T}} & (n+1)=\mathbf{v}(n) \mathbf{v}^{\mathrm{T}}(n) \\
& -\mu(n) \mathbf{v}(n) \mathbf{v}^{\mathrm{T}}(n) \mathbf{x}(n) \mathbf{x}^{\mathrm{T}}(n) \\
& +\mu(n) \eta(n) \mathbf{v}(n) \mathbf{x}^{\mathrm{T}}(n) \\
& -\mu(n) \mathbf{x}(n) \mathbf{x}^{\mathrm{T}}(n) \mathbf{v}(n) \mathbf{v}^{\mathrm{T}}(n) \\
& +\mu^{2}(n) \mathbf{x}(n) \mathbf{x}^{\mathrm{T}}(n) \mathbf{v}(n) \mathbf{v}^{\mathrm{T}}(n) \mathbf{x}(n) \mathbf{x}^{\mathrm{T}}(n) \\
& -\mu^{2}(n) \eta(n) \mathbf{x}(n) \mathbf{x}^{\mathrm{T}}(n) \mathbf{v}(n) \mathbf{x}^{\mathrm{T}}(n) \\
& +\mu(n) \eta(n) \mathbf{x}(n) \mathbf{v}^{\mathrm{T}}(n) \\
& -\mu^{2}(n) \eta(n) \mathbf{x}(n) \mathbf{v}^{\mathrm{T}}(n) \mathbf{x}(n) \mathbf{x}^{\mathrm{T}}(n) \\
& +\mu^{2}(n) \eta^{2}(n) \mathbf{x}(n) \mathbf{x}^{\mathrm{T}}(n)
\end{aligned}
$$

Definindo $\mathbf{K}(n)=E\left[\mathbf{v}(n) \mathbf{v}^{\mathrm{T}}(n)\right]$, tomando o valor esperado de (30), e considerando (i) e (ii), obtém-se

$$
\begin{aligned}
& \mathbf{K}(n+1)=\mathbf{K}(n)-E[\mu(n)][\mathbf{R K}(n)+\mathbf{K}(n) \mathbf{R}] \\
& \quad+E^{2}[\mu(n)]\{\mathbf{R} \operatorname{tr}[\mathbf{R} \mathbf{K}(n)]+2 \mathbf{R} \mathbf{K}(n) \mathbf{R}\} \\
& \quad+E^{2}[\mu(n)] \mathbf{R} \sigma_{\eta}^{2} .
\end{aligned}
$$

Finalmente, a expressão para a curva de aprendizagem é dada por

$$
J(n)=E\left[e^{2}(n)\right]=\sigma_{\eta}^{2}+\operatorname{tr}[\mathbf{R K}(n)] .
$$

\section{Resultados de Simulação}

Nesta seção, são apresentadas simulações numéricas visando verificar a precisão do modelo aqui derivado. Para tal, considera-se um problema de identificação de sistema. A planta desconhecida é dada pelo vetor $\mathbf{w}_{\mathrm{o}}=\left[\begin{array}{lll}1,2 & -0,92,2\end{array}\right.$ $3,1-2,1-2,9]^{\mathrm{T}}$. O sinal de entrada é correlacionado, gaussiano, de média zero e variância $\sigma_{x}^{2}=1,5$, obtido de um processo $\mathrm{AR}(1)$, dado por $x(n)=a x(n-1)+u(n)$, com $a=0,8$ e $u(n)$ sendo um ruído branco gaussiano com média zero e variância $\sigma_{u}^{2}=0,54$. A dispersão de autovalores da matriz $\mathbf{R}$ é $\chi=34,5$. Além disso, um ruído aditivo branco gaussiano $\eta(n)$ com variância $\sigma_{\eta}^{2}=0,1$ é adicionado à saída da planta. Por razões de estabilidade do algoritmo, o valor máximo do passo variável $\mu(n)$ é limitado a 0,01 . Os valores dos parâmetros do algoritmo são $\alpha=0,97, \beta=0,99$ e $\gamma=0,0013$. As Figs. 2, 3, 4 e 5 apresentam os resultados obtidos por simulação Monte Carlo (MC) (média de 200 rodadas independentes) e pelo modelo proposto. Nessas figuras, são comparados simulações e modelo: da evolução do passo variável (Fig. 2), da autocorrelação do sinal de erro (Fig. 3), do comportamento médio do vetor de coeficientes (Fig. 4) e do erro quadrático médio (EQM) (curva de aprendizagem) (Fig. 5). A partir dessas figuras, constata-se o bom casamento entre os resultados de simulação e aqueles obtidos através do modelo proposto.

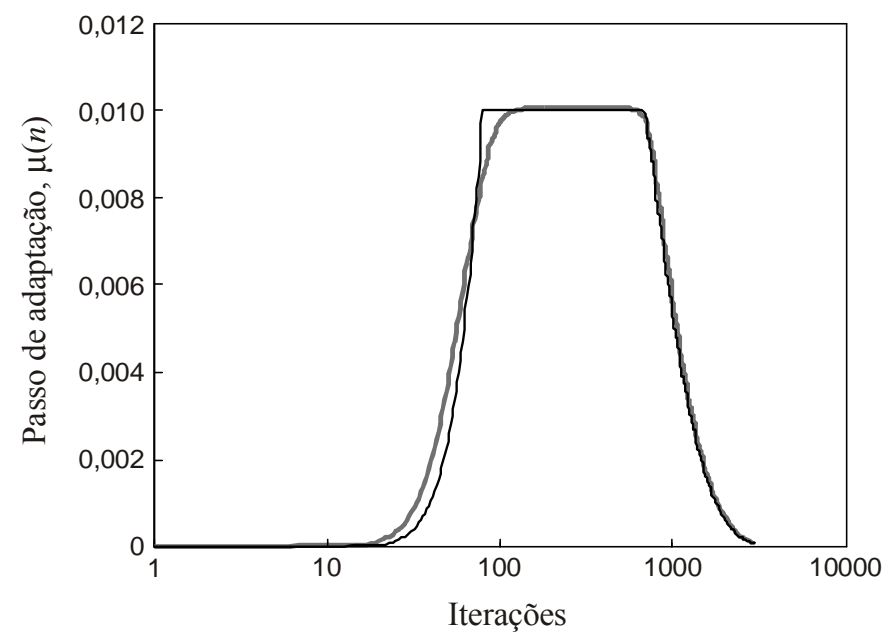

Fig. 2. Evolução do passo variável de adaptação. (Linha cinza) simulação MC. (Linha escura) modelo proposto (10).

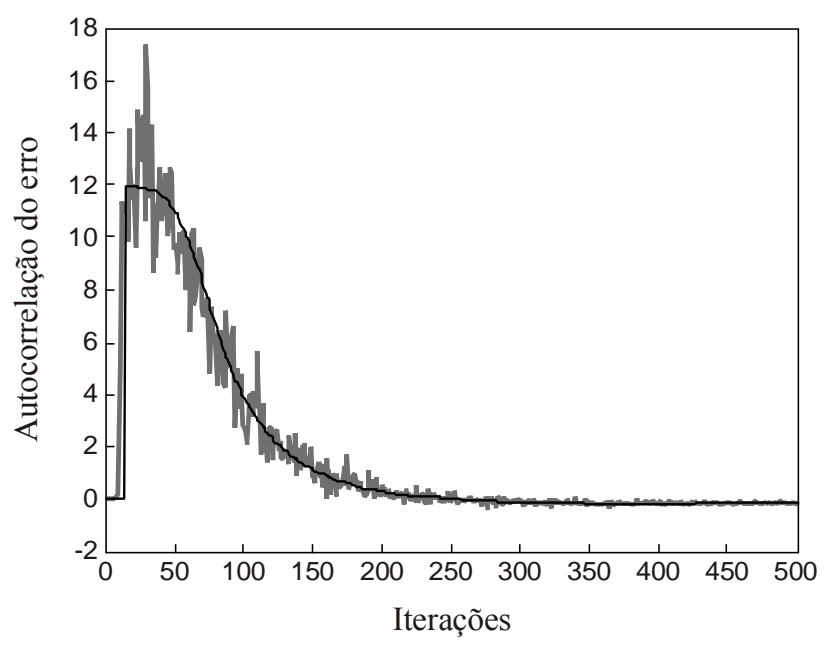

Fig. 3. Evolução da autocorrelação do erro. (Linha cinza irregular) simulação MC. (Linha escura) modelo proposto (20).

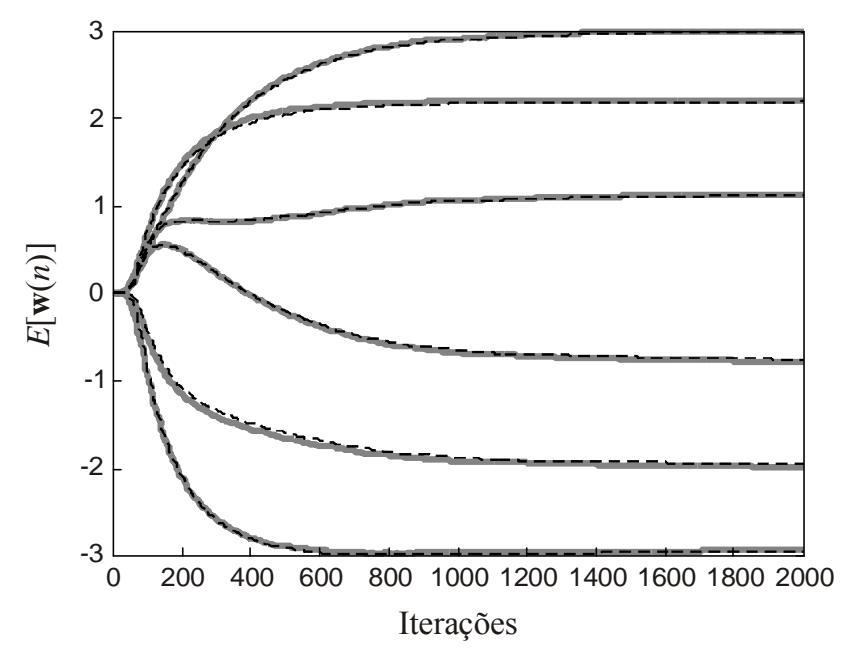

Fig. 4. Comportamento médio do vetor de coeficientes. (Linhas cinzas) simulação MC. (Linhas escuras tracejadas) modelo proposto (8). 


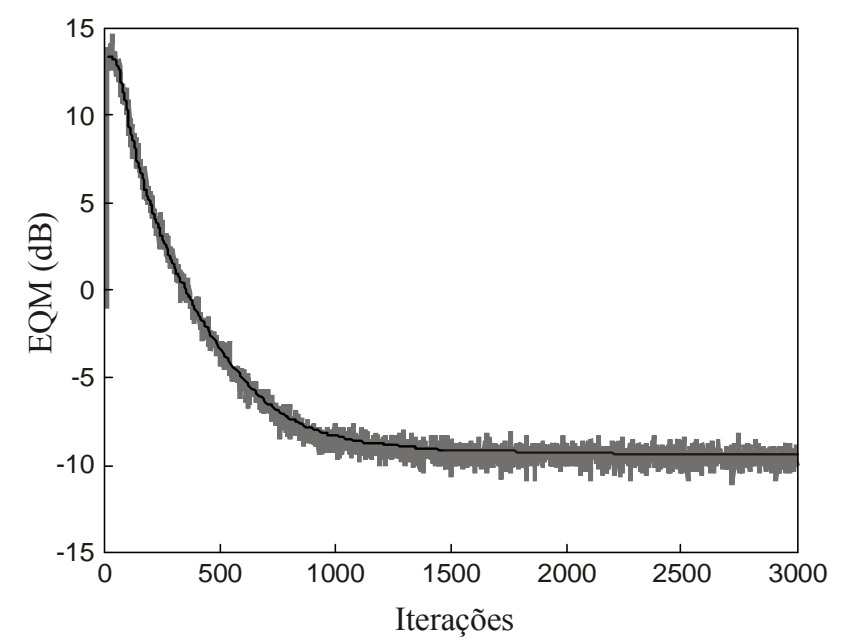

Fig. 5. Curva de aprendizagem. (Linha cinza irregular) simulação MC. (Linha escura) modelo proposto (32).

\section{CONCLUSÕES}

Neste trabalho, foram apresentadas expressões analíticas descrevendo o comportamento médio do vetor de coeficientes e a curva de aprendizagem de um algoritmo VSSLMS baseado na função de autocorrelação do sinal de erro. O modelo foi obtido sem invocar a TI e assumindo adaptação lenta. Resultados de simulações numéricas (para sinais de entrada correlacionados) mostram o bom casamento existente entre simulação $\mathrm{MC}$ e modelo proposto tanto em regime permanente quanto no transitório.

\section{REFERÊNCIAS}

[1] S. Haykin, Adaptive Filter Theory, $4^{\text {th }}$ ed. Upper Saddle River, NJ: Prentice Hall, 2002.

[2] J. C. Richards, M. A. Webster, and J. C. Principe, "A gradient-based variable step-size LMS algorithm," in Proc. IEEE Southeastcon, Williamsburg, USA, Apr. 1991, vol. 2, pp. 1083-1087.

[3] V. J. Mathews and Z. Xie, "A stochastic gradient adaptive filter with gradient adaptive step size," IEEE Trans. Signal Process., vol. 41, no. 6, pp. 2075-2087, Jun. 1993.

[4] A. I. Sulyman and A. Zerguine, "Convergence and steady state analysis of a variable step-size normalized LMS algorithm," in Proc. IEEE Int. Symp. Signal Process. and Its Applications (ISSPA), Paris, France, Jul. 2003, vol. 2, pp. 591-594.

[5] B. Farhang-Boroujeny, "Variable step size LMS algorithm - New developments and experiments," IEE Proceedings - Vision, Image, Signal Processing., vol. 141, no. 5, pp. 311-317, Oct. 1994.

[6] T. J. Shan and T. Kailath, "Adaptive algorithms with an automatic gain control feature," IEEE Trans. Circuits Syst., vol. CAS-35, no. 1, pp. 122-127, Jan. 1988.

[7] J. Okello, Y. Itoh, Y. Fukui, I. Nakanishi, and M. Kobayashi, "A new modified variable step size for the LMS algorithm," in Proc. IEEE Int. Symp. Circuits Syst. (ISCAS), Monterey, USA, Jun. 1998, vol. 5, pp. 170-173.

[8] W-P. Ang and B. Farhang-Boroujeny, "A new class of gradient adaptive step-size LMS algorithms," IEEE Trans. Signal Process., vol. 49, no. 4, pp. 805-810, Apr. 2001.

[9] R. H. Kwong and E. W. Johnston, "A variable step size LMS algorithm," IEEE Trans. Signal Process., vol. 40, no. 7, pp. 1633-1642, Jul. 1992.

[10] I. Nakanishi and Y. Fukui, "A new adaptive convergence factor algorithm with the constant damping parameter," IEICE Trans. Fundamentals, vol. E78-A, no. 6, pp. 649-655, Jun. 1995.
[11] M. H. Costa and J. C. M. Bermudez, "A robust variable step size algorithm for LMS adaptive filters," in Proc. IEEE Int. Conf. Acoust., Speech, Signal Process. (ICASSP), Toulouse, France, May 2006, vol. 3, pp. 93-96.

[12] T. Aboulnasr and K. Mayyas, "A robust variable step-size LMS-type algorithm: analysis and simulations", IEEE Trans. Signal Process., vol. 45, no. 3, pp. 631-639, Mar. 1997.

[13] D. W. Kim, J. H. Hoi, Y. S. Choi, C. H. Jeon, and H. Y. Ko, "A VS-LMS algorithm using normalized absolute estimation error", in Proc. IEEE Digital Signal Processing Applications (TENCON), Perth, Australia, Nov. 1996, vol. 2, pp. 692-697.

[14] Z. Ramadan and A. Poularikas, "A robust variable step-size LMS algorithm using error-data normalization," in Proc. IEEE Southeastcon, Huntsville, USA, Apr. 2005, pp. 219-224.

[15] B. Rohani and K. S. Chung, "A modified LMS algorithm with improved convergence," in Proc. IEEE Singapore Int. Conf. Communication Systems, Singapore, Nov. 1994, pp. 845-849.

[16] D. L. Duttweiler, "Proportionate normalized LMS adaptation in echo cancellers", IEEE Trans. Speech Audio Process., vol. 8, no. 5, pp. 508-518, Sep. 2000.

[17] J. Benesty and S. L. Gay, "An improved PNLMS algorithm," in Proc. IEEE Int. Conf. Acoust., Speech, Signal Process. (ICASSP), Orlando, USA, May 2002, pp. 1881-1884.

[18] S. Gollamudi, S. Nagaraj, S. Kapoor, and Y. Huang, "Set-membership filtering and a set-membership normalized LMS algorithm with an adaptive step size," IEEE Signal Process. Lett., vol. 5, no. 5, pp. 111-114, May 1998.

[19] H. C. Shin, A. H. Sayed, and W. J. Song, "Variable step-size NLMS and affine projection algorithms," IEEE Signal Process. Lett., vol. 11, no. 2, pp. 132-135, Feb. 2004.

[20] J. Benesty, H. Rey, L. R. Vega, and S. Tressens, "A nonparametric VSS NLMS algorithm," IEEE Signal Process. Lett., vol. 13, no. 10, pp. 581-584, Oct. 2006.

[21] Y.-B. Qu, F.-G. Meng, and L. Gao, "A new variable step size LMS adaptive filtering algorithm," in Proc. IEEE Int. Symp. Industrial Electronics, Vigo, Spain, Jun. 2007, pp. 1601-1605. 